\title{
Tendencias y cambio del clima del valle del Mantaro mediante los análisis de índices de Precipitación Efectiva y Temperatura Eficiente
}

\author{
Trends and climate change of the Mantaro valley through the \\ analysis of indicators of effective precipitation and efficient \\ temperature
}

\section{RESUMEN}

Objetivos: La investigación fue para evaluar los cambios y tendencias del clima del valle del Mantaro en función del análisis de sus índices de temperatura eficiente y precipitación efectiva a partir de los valores totales mensuales de precipitación y valores medios mensuales de temperatura, medidos desde el año 1921 hasta el año 2010, para comparar la tendencia de los movimientos seculares con la teoría del calentamiento climático global. Métodos: Para encontrar las tendencias de las precipitaciones y temperatura, se analizaron los índices de precipitación efectiva y temperatura eficiente; luego se determinó el comportamiento interanual de la precipitación y temperatura con sus rangos de variabilidad máximo y mínimo para determinar el tipo de clima en función del coeficiente de variación; las series permitió encontrar las tendencias cíclicas de corta y larga duración mediante el análisis de índices para evaluar la tendencia general. Resultados: Las tendencias observadas en las series cíclicas de larga duración de los índices de temperatura eficiente y precipitación efectiva muestran un comportamiento inversamente proporcional a su movimiento secular, el aumento de la temperatura genera disminución en las precipitaciones y los movimientos seculares muestran estos cambios para la temperatura en cuarenta años y de ochenta años para la precipitación. Conclusiones: Los cambios y tendencias encontradas para el clima del valle del Mantaro muestran un mayor calentamiento y una disminución de la precipitación en las décadas analizadas, siendo los últimos años de la década del 2000 la fase de inversión; por lo tanto se afianza la teoría del calentamiento climático global.

Palabras clave: Clima, cambio climático, variabilidad.

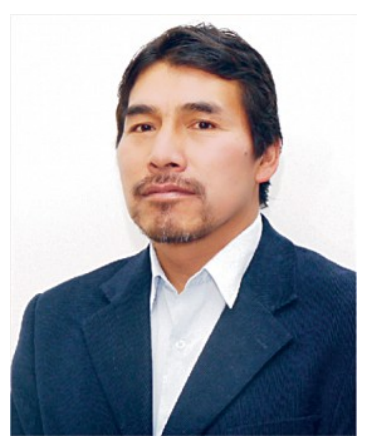

Jacinto Arroyo Aliaga ${ }^{1}$ Instituto Geofísico del Perú

\section{ABSTRACT}

Objectives: The investigation was to evaluate the changes and tendencies of the Mantaro Valley climate in function of the analysis of its indicators of efficient temperature and effective precipitation begining with monthly total values of precipitation and monthly average values of temperature, measured from the year 1921 until the year 2010; also to compare the tendency of the secular movements with the global warming theory. Methods: To find the tendency of precipitations and temperature, the indicators of effective precipitations and efficient temperature were analyzed; then the inter annual behavior of the precipitation and temperature with their ranges of maximum and minimum to determine

1 Investigador adjunto del Instituto Geofísico del Perú en el área de Climatología del Observatorio de Huancayo 
the climate type in function of the coefficient of variation; the series allowed to find the recurrent tendencies of both short and long duration through the analysis of the indicators to evaluate the general tendency. Results: The observed tendencies in the recurrent series of long duration of the indicators of efficient temperature and effective precipitation show us a behavior inversely proportional to their secular movements, the increase in temperature generates decrease in precipitations, and the secular movements show these changes in temperature in forty years and eighty years in precipitation. Conclusions: The changes and trends that were found in the climate of Mantaro Valley show more warming and decrease in precipitation during the analyzed decades, being the last years of the decade of the 2000 the phase of change; therefore the global warming theory is guaranted.

Keywords: Climate, climate change, variability.

\section{INTRODUCCIÓN}

Los cambios climáticos y la variabilidad es una amenaza que afecta a los sistemas naturales y humanos, porque determina el desarrollo económico y la habilidad de muchos países para hacer frente a esta amenaza. El clima siempre ha constituido un factor de preocupación debido a que más del $70 \%$ de los desastres que ocurren en el mundo han estado relacionados con modificaciones del clima y del tiempo.

Esto es el propósito de la investigación, conocer los cambios y las tendencias del clima del Valle del Mantaro, mediante el análisis de los índices de temperatura eficiente y precipitación efectiva a partir de los datos observados de temperatura media horaria mensual y precipitación acumulada mensual, desde el año 1921 hasta 2010.

Los índices resultan ser los indicadores apropiados para observar las tendencias cíclicas y seculares de ambas variables porque muestran el aumento de temperatura y la disminución de la precipitación en el tiempo y evidencian las tendencias globales del calentamiento global que afecta también al Valle del Mantaro.

Dentro de los sistemas de formaciones vegetales del mundo, el valle del Mantaro, por su temperatura media anual de $11,7^{\circ} \mathrm{C}$, y

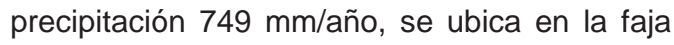
transicional de Bosque Seco Montano Tropical en las partes bajas sureñas y Bosque Húmedo Montano en la provincia de Jauja (1).

Las variaciones en el piso del valle comprendidos entre la laguna de Paca por el norte y Víquez en el sur, muestra una variación climática moderada básicamente por la cantidad de precipitación, siendo relativamente mayor en la zona sur que en el norte, teniéndose la mayor diferencia de lluvias en los meses del verano del valle del Mantaro (2).

La circulación general de la atmósfera es otro factor importante que determina el clima del valle del Mantaro, así tenemos los sistemas de alta presión atmosférica generados por el anticiclón del Atlántico sur y Pacífico sur, que se ubica cerca de las costas de Brasil (alrededor de los $27^{\circ} \mathrm{S}$ ) y Chile (alrededor de los $23^{\circ} \mathrm{S}$ ), estas corrientes oceánicas influyen en las condiciones ambientales de la zona (3).

El paso del sol por el zenit, en su tránsito (aparente) hacia el sur, en el mes de noviembre y su retorno hacia el norte en el mes de febrero origina el aumento de la radiación solar en estos meses debido a que los rayos solares caen más perpendicularmente a la superficie que en otros meses del año (mayo - agosto), este evento hace que todas las variables meteorológicas cambien sucesivamente durante el año y con ello las condiciones del clima(4).

Los valles interandinos de la sierra, se encuentran alterados frecuentemente por su misma posición dentro de las cadenas de montañas, siendo influenciados por su altitud, relieve tan irregular que dan origen a su típico clima de montaña que se modifica y adapta a las ramificaciones orográficas provenientes del océano Atlántico y dan origen a su tiempo invernal o lluvioso (5).

\section{MATERIAL Y MÉTODOS}

El valle del Mantaro, se encuentra en el departamento de Junín en la región central del territorio peruano, tiene una extensión de $75 \mathrm{~km}$, con anchos que varían de $2 \mathrm{~km}$ a $28 \mathrm{~km}$, orientado de NW a SE, entre las latitudes $11^{\circ} 40^{\prime} \mathrm{y}$ $12^{\circ} 10^{\prime} \mathrm{S}$ y los meridianos $75^{\circ} 10^{\prime}$ y $75^{\circ} 22^{\prime} \mathrm{W}$, con altitudes comprendidas entre 3150 msnm y 3400 msnm (figuras 1 y 2). Comprende políticamente a 
las provincias de Jauja, Concepción, Chupaca y Huancayo.

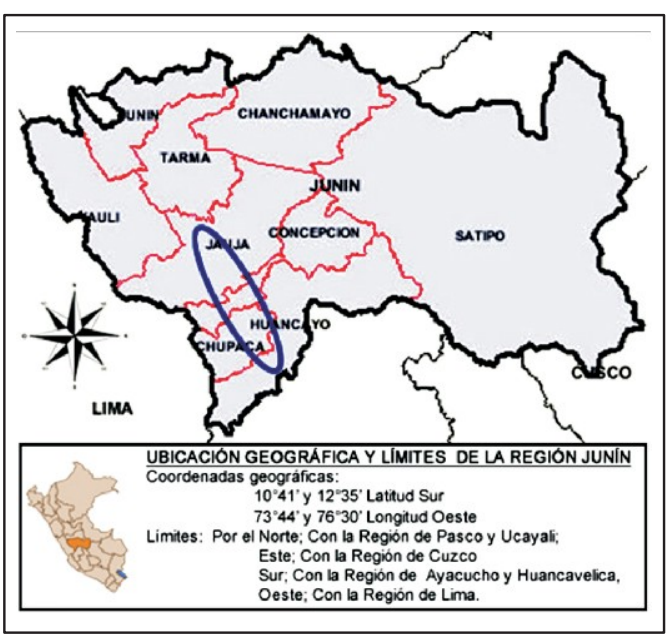

Figura 1. Ubicación geográfica del Valle del Mantaro.

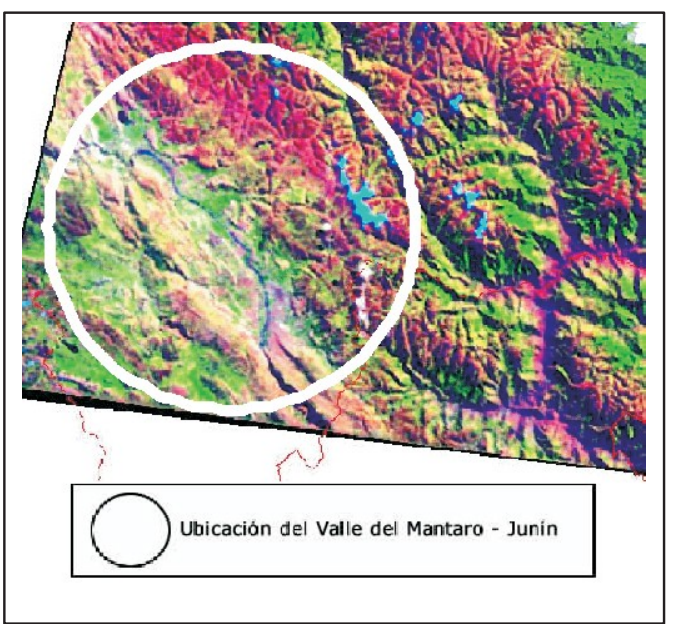

Figura 2. Imagen satelital del Valle del Mantaro.

Fuente: Satélite topex-poseidon

\section{Índices de precipitación efectiva y temperatura eficiente:}

Se han utilizado las siguientes ecuaciones que parte de la influencia de la humedad y de la temperatura para el desarrollo vegetal buscando índices relativos de humedad y temperatura que expresen el grado favorable para la vida de las plantas. $i=1.64\left(\begin{array}{c}t \\ t+12,2\end{array}\right)^{10}$

En donde:

i = índice de precipitación efectiva para un mes

$\mathrm{f}=$ precipitación pluvial

$\mathrm{t}=$ temperatura media mensual

La temperatura eficiente se considera como una función de temperatura y evaporación, se calculamediantelasiguienteecuación:

$i^{\prime}=\frac{9 t}{20}$

Variabilidad:

$\sigma=\frac{\sum\left(x_{i}-\bar{x}\right)^{2}}{n}$

Dónde:

$\mathrm{xi}=$ un solo valor

$x=\frac{\sum_{i=1}^{n} x_{i}}{n}$

$\mathrm{n}$ = número de casos

$\Sigma$ = Suma de todos los valores

Las variabilidades de la precipitación correspondientes a distintos meses pueden compararse entre sí, si se expresa la desviación típica en tanto por ciento de la media ( $\mathrm{CV}=$ coeficiente de variabilidad)

$$
C V=\frac{\sigma}{\bar{x}} \times 100(\%)
$$

Tendencia:

Se encuentra la tendencia analizando la serie de tiempo de los (VTM) de precipitación y (VMM) de temperatura, basadoenlasiguiente ecuación

$\mathrm{Y}=\mathrm{T} \times \mathrm{C} \times \mathrm{S} \times \mathrm{I}$

Donde:

$\mathrm{Y}=$ Valores de las variables

T = Movimiento secular o de larga duración

$\mathrm{C}=$ Movimiento cíclico

$\mathrm{S}=$ Movimiento estacional

I=Movimientosirregularesoalazar 
Se ha recurrido a las ecuaciones de la Transformada Rápida de Fourier para encontrar las periodicidades cíclicas o estacionales de las variables en estudio y para predecir el comportamiento en el futuro del clima del Valle del Mantaro se ha recurrido al modelo armónico quetiene comoecuación:

$\mathrm{T}(t)=C_{0}+\sum_{n=1}^{\infty} C_{n} \operatorname{sen}\left(\frac{2 \pi}{T_{n}} t+\Phi_{n}\right)+\sigma$

Donde:

$C_{n}=$ amplitud

$\Phi_{n}^{n}=$ fase

$T_{n} \quad=$ periodo

$t_{0} \quad=$ eslaépoca de inicio del modelo,

$\mathrm{C}_{0} \quad=$ valormedio

$\sigma=$ error

\section{RESULTADOS}

\section{Caracterización del tipo de clima del Valle del Mantaro}

La figura 3, muestra la distribución mensual de la temperatura en el valle del Mantaro, como resultado de la caracterización en función del índice de temperatura eficiente y la figura 4 , muestra la distribución mensual de la precipitación en función del análisis de la precipitación efectiva. Además estos resultados se asocian con los límites máximos permisibles de variabilidad en función de cada mes.

Ambas figuras se comparan con el promedio multianual que divide en dos tipos de comportamiento en función de la temperatura y precipitación (líneas entrecortadas rectas en ambosgráficos.

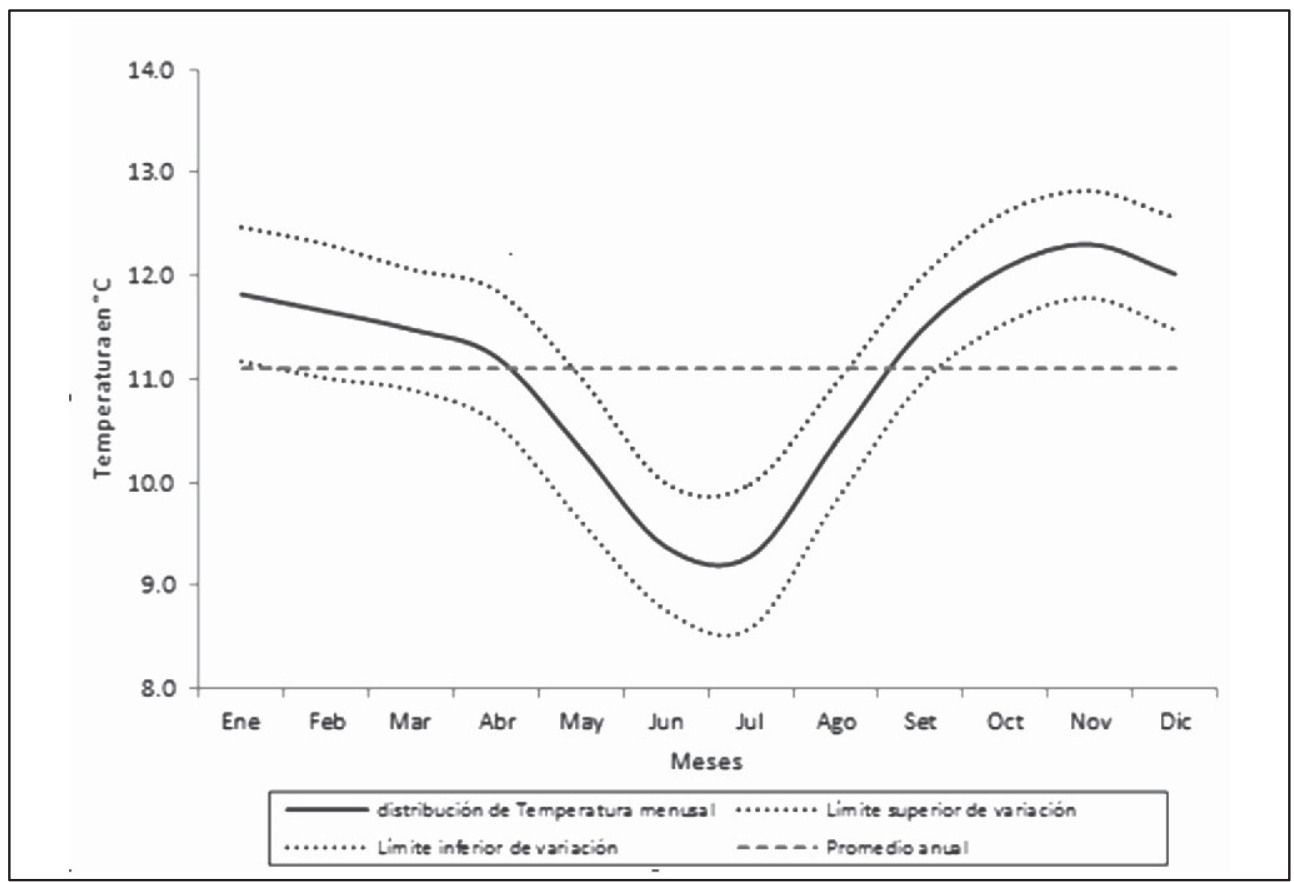

Figura3: Distribución mensual de temperatura en el Valle del Mantaro 1921 - 2010 


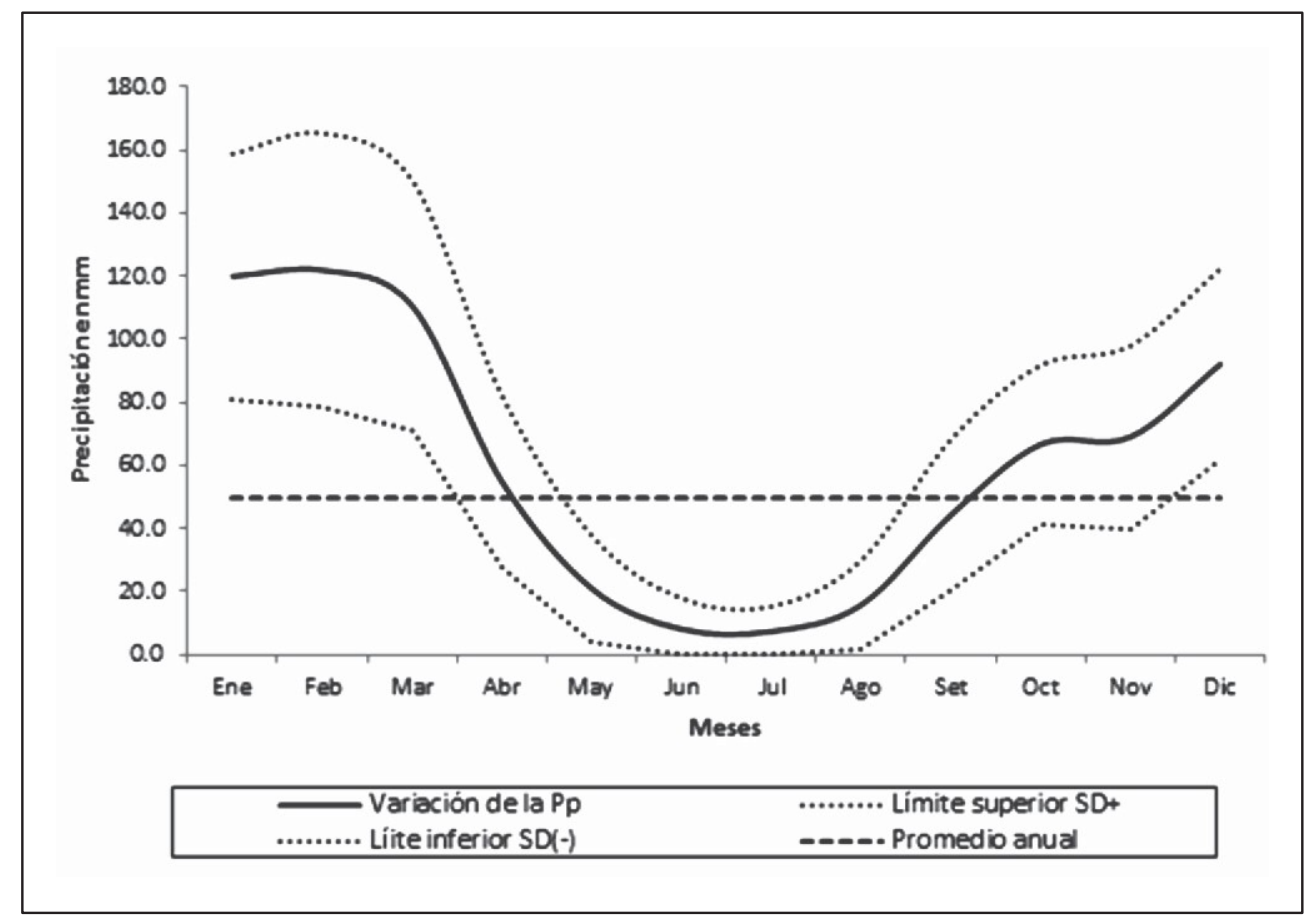

Figura 4:Distribución mensual de la precipitación en el Valle del Mantaro 1921 - 2010

Se han utilizado las siguientes ecuaciones que parte de la influencia de la humedad y de la temperatura para el desarrollo vegetal buscando índices relativos de humedad y temperatura que expresen el grado favorable para la vida de las plantas.

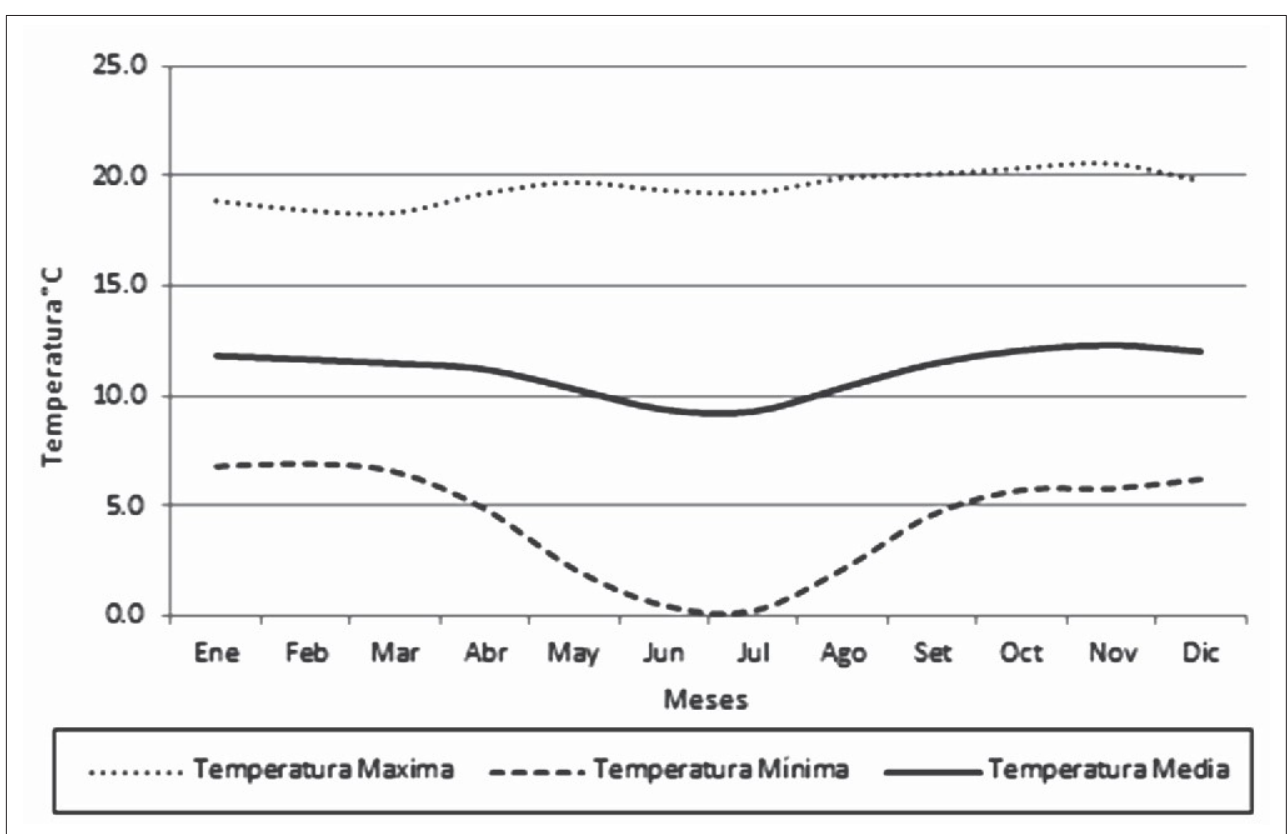

Figura 5. Distribución de la variabilidad de temperatura del Valle del Mantaro, 1921 - 2010. 
La tabla 1, muestra la comparación de frecuencias de precipitación por décadas en el valle del Mantaro, los intervalos de clase se divide cada $50 \mathrm{~mm}$ de precipitación empezando desde $0 \mathrm{~mm}$ hasta $250 \mathrm{~mm}$ como valor máximo que se ha registrado históricamente. Además se presenta el promedio interdecadal de frecuencia así como la desviación estándary la mediana.

\section{Variaciones del clima del valle del Mantaro, basado en los índices de precipitación efectiva (ipe) y temperatura eficiente (ite)}

La figura 6, muestra la tendencia por década de la variación de índices de precipitación efectiva y temperatura eficiente del valle del Mantaro, desde la década 1921 - 1930, hasta la década 2001 - 2010.

Tabla 1. Frecuencias de precipitación por década en el Valle del Mantaro

\begin{tabular}{ccccccccccc}
\hline Intervalos & Marca de & $21-$ & $31-$ & $41-$ & $51-$ & $61-$ & $71-$ & $81-$ & $91-$ & $01-$ \\
de clase & clase & 30 & 40 & 50 & 60 & 70 & 80 & 90 & 00 & 10 \\
\hline-50 & 25 & 55 & 57 & 55 & 62 & 55 & 57 & 57 & 57 & 61 \\
$51-100$ & 75 & 41 & 44 & 38 & 28 & 41 & 36 & 30 & 35 & 40 \\
$101-150$ & 125 & 20 & 12 & 21 & 22 & 18 & 18 & 21 & 25 & 15 \\
$151-200$ & 175 & 3 & 5 & 6 & 8 & 6 & 8 & 8 & 3 & 4 \\
201 & 1 & 2 & 0 & 0 & 0 & 1 & 4 & 0 & 0 \\
\hline & 225 & & & & & & & & & \\
\hline
\end{tabular}

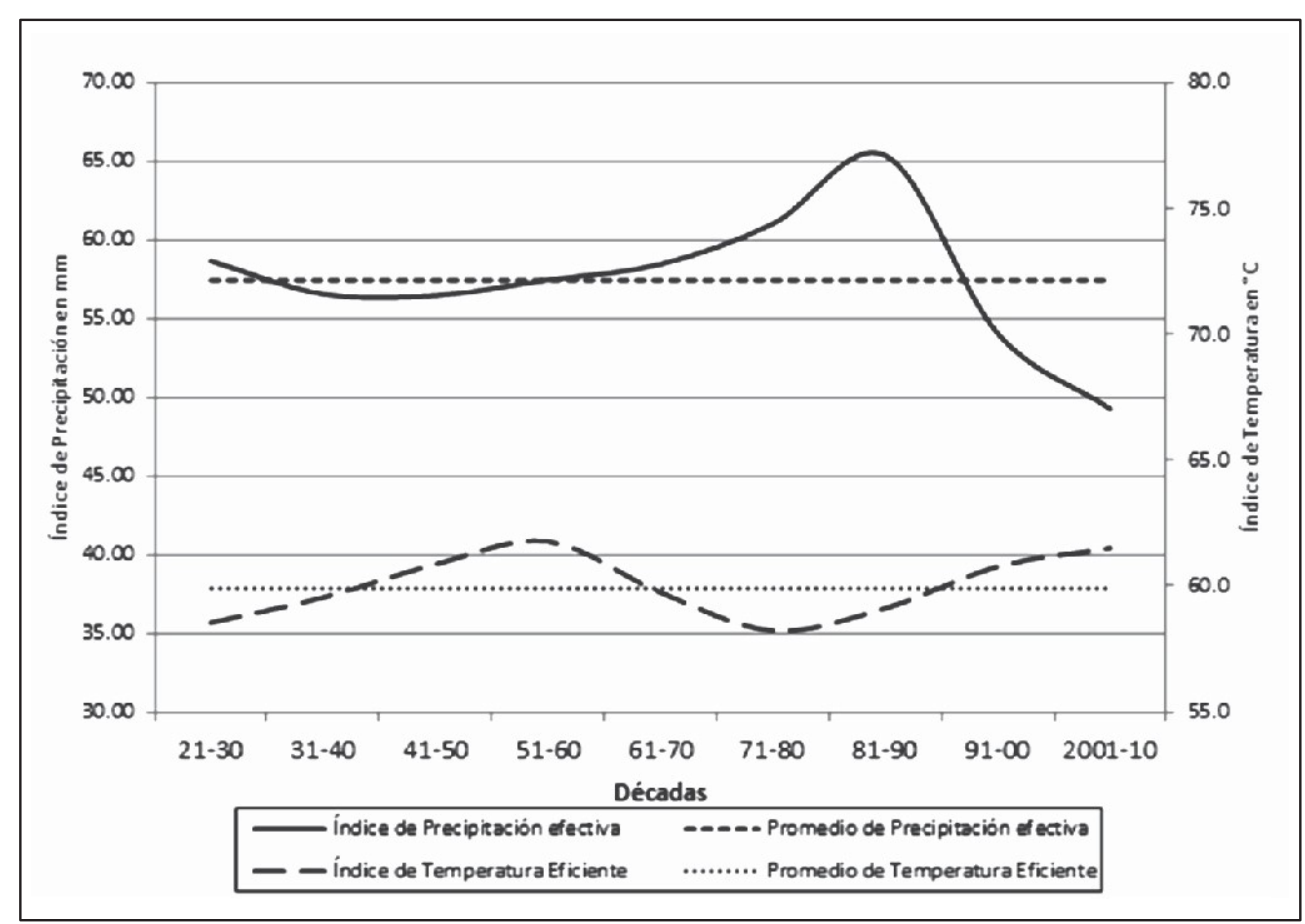

Figura 6.Variación de los índices de recipitación efectiva y temperatura eficiente del Valle del Mantaro 1921 - 2010 


\section{Variabilidad de precipitación y temperatura:}

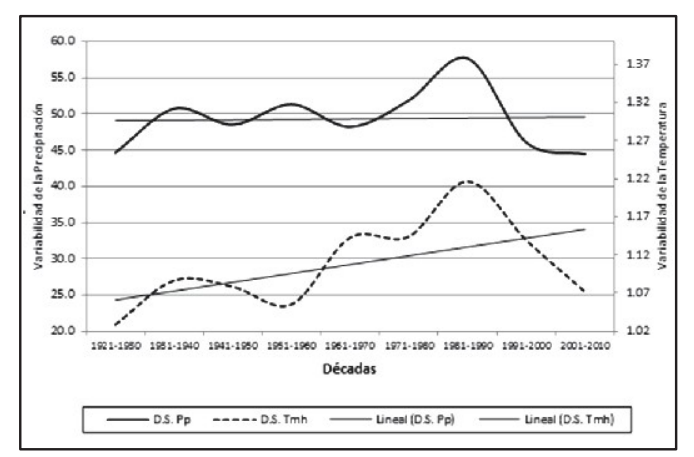

Figura7. Variabilidad de la precipitación y temperatura del Valle del Mantaro 1921 - 2010

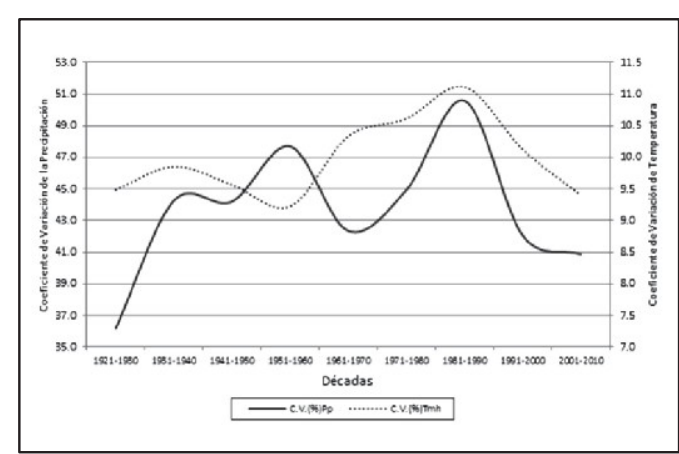

Figura 8. Coeficiente de variación de la precipitación y temperatura del Valle del Mantaro 1921 - 2010

\section{Análisis de tendencias:}

La figura 9, muestra la tendencia longitudinal en el tiempo de la relación entre la precipitación efectiva y la temperatura eficiente en periodo de corta duración (a) y en periodos de serie de larga duración. Se presenta estas figuras mediante el análisis de promedios móviles de corta duración cada 5 años y de larga duración cada 10 años con el fin de evitar la influencia de la dispersión y tener un mejor ajuste en la tendencia de comportamiento.

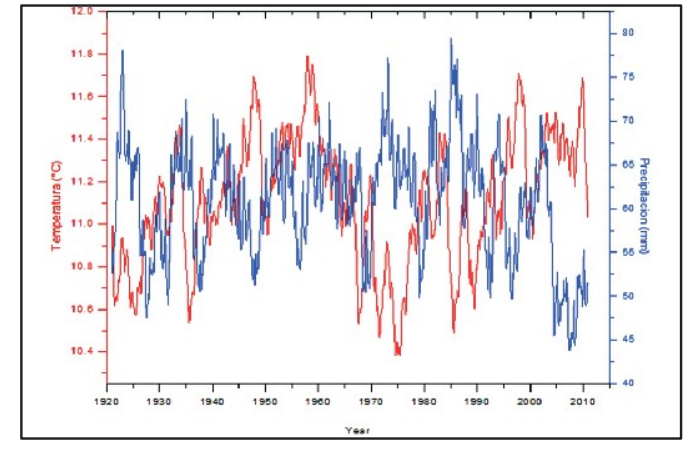

a) Serie de corta duración.

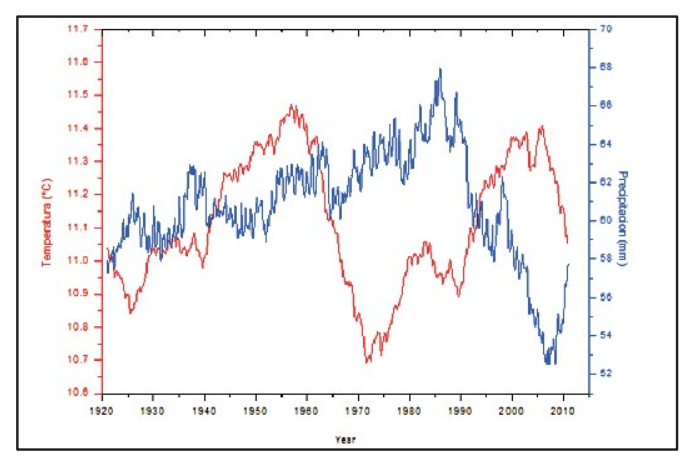

b) Serie de larga duración.

Figura 9. Comparación entre series de corta y larga duración de la temperaturay precipitación. 1921-2010.

La figura 10, muestra la tendencia de larga duración de la precipitación efectiva y temperatura eficiente ajustada mediante el método estadístico de promedios móviles cada 20 años. El fin es eliminar las influencias estacionales de la variabilidad de la precipitación y temperatura para tener un mejor ajuste de comportamiento cíclico de larga duración.

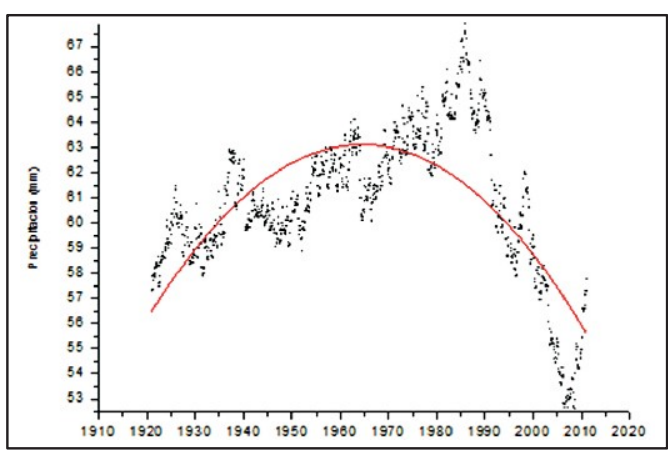

a) Precipitación 


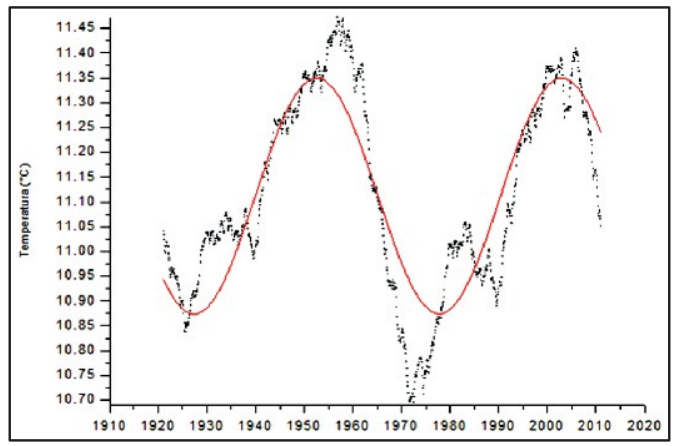

b) Temperatura

Figura 10. Análisis del comportamiento de series de larga duración de la temperaturay precipitación. 1921-2010

La figura 11, muestra las periodicidades cíclicas encontradas de la temperatura eficiente y precipitación efectiva por décadas desde la década 1921 - 1930 hasta la década 2001 2010. Analizado mediante las ecuaciones de la Transformada Rápida de Fourier y poder predecir el comportamiento del cima futuro.

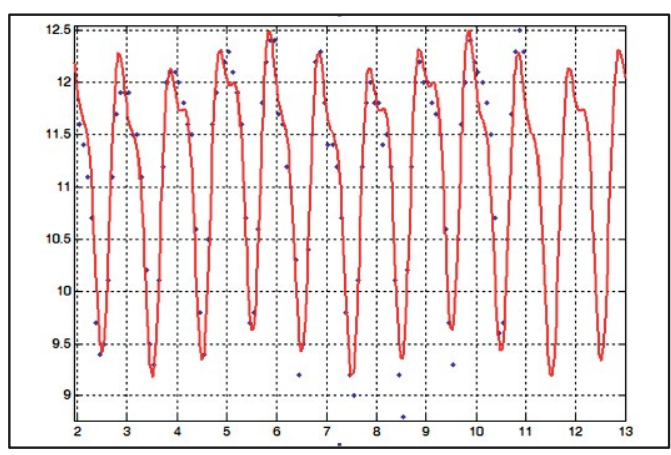

a) Temperatura

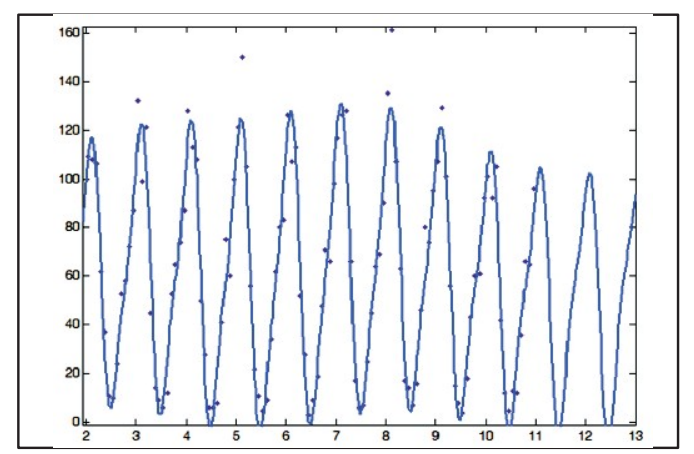

b) Precipitación

Figura 11.Estimación de periodicidades de la temperatura y precipitación 1921-2010

\section{DISCUSIÓN}

El clima del Valle del Mantaro por sus valores de índice climático basado en la precipitación efectiva y temperatura eficiente tiene un tipo de clima ( $\left.C^{\prime}\right)$ que corresponde a las características de sub húmedo y micro térmico de carácter frío, por sus valores de 59,47 y 60,00 respectivamente (figura 3). Por su comportamiento de las precipitaciones, se clasifica en sub húmedo debido a su comportamiento hídrico.

Durante el año se presenta dos épocas muy típicas (Figura 4), un periodo seco (estiaje), comprendido entre los meses de abril - setiembre (seis meses) y otra estación húmeda (lluvioso), que comprende los meses de octubre-marzo. Estas características presentes en la temperatura están muy asociadas con el movimiento de traslación de la Tierra alrededor del Sol.

En julio la Tierra se encuentra alejado al Sol (afelio), por consiguiente la cantidad de rayos solares que están más cerca al horizonte inciden oblicuamente, produciendo menor calentamiento, además de que los rayos solares deben pasar a través de una capa atmosférica más gruesa. En noviembre en donde el Sol se va acercando a la Tierra se presentan las temperaturas más altas, por efectos de la transparencia de la atmósfera que tiene gran efecto sobre la cantidad de radiación que llega a la superficie del Valle. En enero la Tierra se encuentra cerca al Sol (perihelio) se debería tener mayor temperatura pero su disminución se debe más a la cantidad de nubes y turbidez ambiental presente en esta temporada que hace que recibamos menos radiación directa.

Las precipitaciones de lluvia y las formaciones de nubes son traídas por el movimiento de masas de aire del sureste, este y noreste que vienen cargados de humedad como consecuencia de la inestabilidad atmosférica producto de la baja presión de estos meses de octubre - marzo, son inducidos a raíz del calentamiento por la alta insolación.

En la estación templada y húmeda se registran las temperaturas más altas en el mes de noviembre con $20,5^{\circ} \mathrm{C}$ en promedio (Figura 5). $\mathrm{Y}$ en los meses de enero, febrero se registran las temperaturas mínimas más altas debidas principalmente a los continuos días de nubosidad que provienen de las masas húmedas continentales. 
Con respecto a las variaciones del clima del valle del Mantaro, basado en los Índices de Precipitación Efectiva(IPE) y TemperaturaEficiente (ITE), observados en la figura 5 y cuadro 1, muestra que en la década de los años 20 se tıvo un IPE de 58,88 con un incremento de $(\Delta=0,30)$ sobre su promedio de 58,58, por lo sus características climáticas esta década fue normal, con 20 meses de frecuencia de Iluvia comprendidos entre $100 \mathrm{~mm} 150 \mathrm{~mm}$ y 41 meses entre $50 \mathrm{~mm} 100 \mathrm{~mm}$, presentándose solamente tres eventos superior a los $150 \mathrm{~mm}$ y un evento superior a los $200 \mathrm{~mm}$. Estos cuatro eventos superiores a los $150 \mathrm{~mm}$, hace que esta época sea ligeramente más húmeda que las condiciones presentes en el promedio.

Esta etapa también nos indica que se encuentra en una década final del proceso de mayor humedad registrada en el tiemno. El ITE de 60,42 , con un incremento de $\quad(\Lambda=0,45)$ sobre su promedio de 59,97, también nos manifiesta un década sustancialmente más cálido que el promedio, tendiendo a ser tener un clima más templado por su temperatura.

La variabilidad de precipitación y temperatura que se muestra en la figura 7, presenta para las precipitaciones un incremento en razón de

$\left(\frac{\Delta P^{\prime}}{\Delta t}=0,19\right)_{Y \mathrm{de}}\left(\frac{\Delta T}{\Delta t}=0,18\right)$

para la temperatura. Además se observa que la menor variabilidad de la precipitación se presenta en la década de los años 20, 60 y 90 y tendiendo a disminuir en la década del 2000. La variabilidad se incrementa en la década de los años 30,50 y 80, siendo esta última década donde se presentó las mayores variaciones de lluvia como se observa en la figura 6. También se observa que luego de presentarse una década de menor variabilidad le prosigue otra década de variabilidad alta. Las líneas de tendencias para ambos son ascendentes.

Con respecto al análisis de tendencias que se observa en la figura 9, indica el comportamiento inverso que tiene las precipitaciones con respecto a la temperatura. En la serie de corta duración dichos comportamientos no son significativos pero en la serie de larga duración que se muestra en la figura 10 , se observa el comportamiento secular cíclico de ambas variables en el tiempo. Para la precipitación existe un ciclo de larga duración mayor a 80 años, mientras que la temperatura presenta dos ciclos en periodos de 40 años.
Las periodicidades cíclicas presentadas en la figura 11, muestran las tendencias en el largo plazo de la temperatura y la precipitación, donde se observa que la temperatura continuará subiendo a razón de $\left(\ddot{A}=0,0017^{\circ} \mathrm{C} / a n ̃ o\right)$ y la precipitación tenderá a disminuir a razón de $(\ddot{A}=$ $-0,13 \mathrm{~mm} / \mathrm{año}$ ). Estas tendencias no difieren de las proyecciones mundiales del cambio climático en donde se afirma que la precipitación disminuirá y la temperatura aumentará para la región de Sudamérica. Demostrando que los índices son los indicadores más apropiados para analizar dichas tendencias. 


\section{REFERENCIAS BIBLIOGRAFICAS}

1. Holdrich LR. Life Zone Ecology - Tropical Science Center. San José: Ediciones del Instituto Ecológico de CR; 1967.

2. Tosi JA. An Ecological Model for the Prediction of Carbon Offsets by Terrestrial Biota Tropical Science Center. Occasional Paper No. 17.34p. San José: Ediciones del Instituto Ecológico de CR; 1997.

3. Senamhi, El Sol y la Radiación Solar. Lima: Publicaciones de manuales meteorológicos; 2005.

4. Valdivia R. Meteorología General. Lima: Ediciones San Marcos; 1999.

5. Arroyo J, Rosales D. Análisis y Modelamiento de la Serie Temporal de la media mensual de temperatura en el Observatorio de Huancayo: Lima: Ediciones Nuevo Horizonte; 2004.

6. Barry R, Chorley R. Atmósfera, Tiempo y Clima. Barcelona: Ediciones Omega; 1995.

7. Gianibelli J. Efectos Solar y Lunar en la Intensidad Total Determinada en el Observatorio Geomagnético de las Acacias, IV Jornadas Geológicas y Geofísicas Bonaerenses. Buenos Aires: Ediciones UBA; 1995.

8. PaluzieA. Las Maravillas delCielo. Barcelona: Ediciones Danae; 1970

9. Rector T, Lockwood J. Blazing Sunspots Studying the Active Sun, Teacher Leaders in Research Based Science Education. Tucson: Ediciones Mc Hill; 2001.

Correo electrónico:

jarroyox@gmail.com 